\title{
Urbane Populärkultur als Bewertungspraxis und -ressource
}

\author{
Zum normativen Potential populärkultureller Inszenierung und \\ diskursiver Aneignung urbaner Räume
}

\begin{abstract}
The essay argues that various forms of urban popular culture function as key place-making practices in that they medially and discursively prefigure the perception and evaluation of urban spaces. Using examples from popular music - Jay- $Z$ and Alicia Keys' "Empire State of Mind" - and street art - in this case the notorious Banksy - we exemplify potential functions of urban popular culture as a place-making practice: The evaluation of urban spaces, we argue, functions both by means of a discursive staging, which - often in strongly normative ways offers recipients patterns and templates of evaluation, and through various practices of interactively appropriating urban spaces, which again shape the perception and evaluation of these spaces. Also taking into account the discourse about urban popular culture and its evaluation as a practice of distinction (sensu Bourdieu) in the generation and propagation of different urban habitus, the essay concludes that urban popular culture functions as both a practice of and a resource in the negotiation of established or emergent notions of 'urbanity'.
\end{abstract}

Was wir über urbane Räume wissen bzw. was wir darüber zu wissen glauben, ist in der Regel erst in zweiter Linie das Resultat eigener Erfahrungen in bzw. mit diesen Räumen. Vielmehr sind unsere Ideen über urbane Räume - und wir meinen hier ganz konkrete wie z.B. Berlin, London, New York, Mexico City, Tokio oder Moskau - in einem nicht unerheblichen Maße medial und diskursiv vorgeprägt. Was wir ,wissen', wissen wir nicht selten aus Zeitungen, aus Reiseführern, aus dem Fernsehen, aus Kinofilmen, aus Songs, aus Romanen, aus Computerspielen oder aus anderen populärkulturellen Ausdrucksformen, die urbane Räume repräsentieren und auf diese Weise unsere Wahrnehmung und damit häufig auch unsere Bewertung eben dieser Räume vorstrukturieren (vgl. Carney 2003a: 212-213).

Ausgehend von der Annahme, dass die tatsächliche Bewertung urbaner Räume durch individuelle und kollektive Akteure in der Regel über populärkulturelle Ausdrucksformen ,vorgeformt' wird, versucht unser Beitrag zu beschreiben, wie sich die ,Bewertung ' urbaner Räume in verschiedenen Manifestationen urbaner Populärkultur als Form des Place-Making verstehen lässt.

$\mathrm{Zu}$ diesem Zweck wirft unser Beitrag einen Blick sowohl auf verschiedene Formen der repräsentationalen Inszenierung urbaner Räume (wir meinen damit 
beispielsweise Filme oder Popsongs, in denen urbane Räume diskursiv konstruiert werden), als auch auf unterschiedliche Praktiken der interagierenden Aneignung urbaner Räume (hiermit meinen wir etwa Formen der performativen Auseinandersetzung mit ebendiesen Räumen wie z.B. Graffiti oder Street Art); wir möchten so deren Wirkungs- und Funktionspotentiale aufzeigen. Die beiden genannten Arten der populärkulturellen ,Beschäftigung‘ mit urbanen Räumen lassen sich vielleicht am ehesten durch ihren Grad der mediacy - also ihrer ,Mittelbarkeit' bzw. medialen ,Vermitteltheit ${ }^{\text {‘ }}$ - voneinander unterscheiden (vgl. hierzu Gurr/Butler 2011: 150-151.). Diese relationale Kategorie erlaubt es nämlich, Formen und Funktionen urbaner kultureller Ausdrucksformen entlang einem Kontinuum zwischen direkten bzw. unmittelbar performativen und indirekten bzw. medial vermittelten Formen der Auseinandersetzung mit urbanen Räumen zu klassifizieren. Zu den ,direkten“ Formen gehören dabei etwa Formen der Performance-Kunst, die die architektonischen und infrastrukturellen Gegebenheiten, Begrenzungen und Möglichkeiten der Stadt ausloten, indem sie geome-trisch exakte und technologisch-funktionale Formen mit dem menschlichen Körper konfrontieren und durch den Kontrast von Lebendigem und ,lebloser ${ }^{\star}$ gebauter Umwelt ${ }^{1}$ den urbanen Raum re-semantisieren und neu perspektivieren. Weitere unmittelbare Formen der Aneignung urbaner Räume wären etwa spezifisch ,urbane‘ Sportarten wie Skateboarding, BMX oder Parcours, aber auch - wie bereits erwähnt - Graffiti und anderen Formen der Street Art. Stärker ,vermittelte‘ Formen der Auseinandersetzung mit dem urbanen Raum wären etwa fiktionale oder nicht-fiktionale ,Texte“ (im weitesten Sinne) - von Stadtführern und Nachrichtensendungen bis zu Stadtromanen und Spielfilmen. Solche medialen Repräsentationen artikulieren nicht nur bestimmte Perspektiven auf die Stadt, sondern inszenieren ggf. auch utopische oder dystopische Szenarien, ,mögliche (Alternativ-)Welten', die (hypothetische) Konsequenzen fortschreitender Urbanisierung und anderer Entwicklungen bewusst machen. Die Stadt ist also häufig nicht nur Ort, sondern auch Thema urbaner kultureller Ausdrucksformen (vgl. hierzu genauer Gurr/Butler 2011: 150 und Gurr 2010: 253254).

Anhand einer auf anglo-amerikanische Kulturräume fokussierten Untersuchung ausgewählter Beispiele (New York und London) soll im Folgenden der Stellenwert populärkultureller Formen der Repräsentation und Aneignung urbaner Räume als kulturelle und symbolische ,Ressource' in individuellen und kollektiven Selbstbildungs- und Abgrenzungsprozessen herausgearbeitet wer-

1 Eine faszinierende Geschichte des Verhältnisses von Stadt und menschlichem Körper findet sich in Richard Sennetts Flesh and Stone: The Body and the City in Western Civilization (1974). 
den. Anhand dieser Analyse, so unsere Annahme, lässt sich dann auch das Potential dieser populärkulturellen Praktiken und Ausdrucksformen in Prozessen der Bewertung urbaner Räume nachzeichnen. Dabei gehen wir davon aus, dass urbane Populärkultur sowohl Bewertungsressource als auch Bewertungspraxis sein kann (wenn sich diese beiden Kategorien überhaupt voneinander trennen lassen).

In einem zweiten Schritt diskutiert der Beitrag außerdem die Formen und Funktionen der Diskursivierung der zur Rede stehenden populärkulturellen Praktiken und Ausdrucksformen (etwa in Form von Filmen über Street Art und - auf einer weiteren Meta-Ebene - deren Kommentierung durch verschiedene Akteure). Wir sind nämlich davon überzeugt, dass auch das, Reden über urbane Kultur‘ eine Form der Bewertung urbaner Räume darstellen kann, denn die in diesen Diskursen zu beobachtenden Strategien und Taktiken der Evaluation urbaner Populärkultur (als ,Subversion“ oder ,Ausverkauf‘, als ,alternativ‘ oder ,mainstream') führen letztlich (je nach Perspektive) ebenso zu einer Auf- bzw. Abwertung urbaner Räume, die stets, so lässt sich zumindest vermuten, mit dem Ziel der „Maximierung der symbolischen Distinktionsgewinne“ (Bourdieu 1989: 398) einhergeht. Diese Praxis der Diskursivierung, verstanden als ,Kommunikation über urbane Kommunikation‘, mag damit als Ausdruck eines spezifisch ,urbanen Habitus' zu verstehen sein. ${ }^{2}$

Ausgehend von diesen Überlegungen zu urbaner Populärkultur als Form „urbaner Kommunikation“ - verstanden sowohl als Kommunikation in urbanen Kontexten als auch als Kommunikation über urbane Räume und Urbanität skizziert der Beitrag abschließend den Zusammenhang zwischen populärkulturellem Bewertungsdiskurs und der Konstitution von Urbanität als Differenzkategorie und postuliert auf dieser Grundlage die Entkopplung von Urbanität vom physischen Raum der Stadt.

\footnotetext{
2 Der Forschungskontext des vorliegenden Beitrags ist ein gemeinsames Forschungsprojekt zu urbaner Populärkultur, das wir an der Universität Duisburg-Essen entwickelt haben. Das Projekt ist Teil eines interdisziplinären Verbundprojekts zum Thema „Urbanität und urbane Kommunikation“ an dieser Universität, welches wiederum in den dortigen Profilschwerpunkt „Urbane Systeme“ eingebettet ist.
} 


\section{Zur medialen Präfiguration urbaner Räume in der Populärkultur}

Bereits im Jahre $1966^{3}$ schlägt der amerikanische Kulturgeograph Peter Gould das Konzept der ,mental maps‘ vor, mit dem er die mediale Vorprägung unserer Wahrnehmung von Landschaften und Regionen $\mathrm{zu}$ fassen versucht. Er argumentiert, dass die Wahrnehmungen bestimmter Orte nicht zuletzt das Ergebnis der von Menschen aus den verschiedensten Kanälen - u.a. Zeitungen und Zeitschriften, Bücher, Filme oder das Fernsehen - aufgenommenen Informationen über diese Orte seien (vgl. Carney 2003a: 212; Gould 1966; Gould/White 1974). Goulds Beobachtung scheint zu Beginn des 21. Jahrhunderts in Anbetracht einer fortschreitenden Medialisierung der Gesellschaft nichts an Aktualität verloren zu haben. Im Gegenteil: Sie ist vermutlich zutreffender als je zuvor. Denn in der Tat ist unser kulturelles Wissen, und zwar nicht nur das über Landschaften und Regionen, in hohem Maße medial präfiguriert, und diese Präfiguration ist nicht zuletzt Grundlage unserer ,Vor-Urteile‘ - hier gemeint in einem wertfreien Sinne - über Menschen, Kulturen, Institutionen und/oder Orte.

Goulds Konzept der ,mental maps‘ und dessen Weiterverarbeitungen im kulturgeographischen Diskurs seit 1966 helfen dabei, diesen (zunächst nur postulierten) Zusammenhang zwischen populärkulturellen Ausdrucksformen und der Bewertung urbaner Räume etwas genauer zu konturieren. So beschreibt beispielsweise George O. Carney im Anschluss an die Ideen Goulds, wie wir unsere Ersterfahrung an einem Ort strukturieren, diesen Ort bzw. dessen Wahrnehmung in unser je individuelles Wissensgefüge einbetten und ihn auf diese Weise sozusagen mit Sinn ,ausstatten':

[P]ersons entering a place for the first time search for cues with which to assign meaning to a place. Prior knowledge of a place might interfere with an objective appraisal by clouding or masking cues, but stereotypes also provide useful frames of reference that can be modified by careful observation. (Carney 2003a: 213)

Was Carney hier zu fassen versucht, ist eben jenes Zusammenspiel zwischen medialer Vorprägung und der tatsächlichen Wahrnehmung im Sinne einer Primärerfahrung: Die Komplexität des Ortes wird reduziert durch die Konzentration auf ,cues` - Schlüsselreize, aus denen sich Bedeutung generieren lässt - und

3 Interessanterweise wird der Begriff „mental map“ im Zusammenhang mit der Wahrnehmung von Städten bereits in Kevin Lynchs einflussreicher Studie The Image of the City von 1960 verwendet. Der Begriff „mental map“ taucht zwar in Lynchs Buch nur an einer Stelle auf (vgl. ebd.: 88); das Konzept ist jedoch bereits zentral. 
,stereotypes‘, die einen ,frame of reference‘, einen Bezugsrahmen zur Strukturierung der Wahrnehmung zur Verfügung stellen. Gleichermaßen wird dieser Prozess der Wahrnehmungsstrukturierung durch ,Altbekanntes“ (,prior knowledge“) empfindlich ,gestört‘; Altbekanntes, das möglicherweise den unterschiedlichen medialen Inszenierungen eben jenes Ortes entstammt, den wir zum ersten Mal aufsuchen (und manchmal dennoch schon zu kennen glauben).

Gerade populärkulturelle Praktiken und Ausdrucksformen, so unsere These, spielen aufgrund ihrer massenhaften Verbreitung und ihrer emotionalaffektiven Dimension (vgl. insb. Grossberg 1992: 79-87) eine gewichtige Rolle in diesem Prozess der Aushandlung von Bedeutung und der Strukturierung von Wissen; sie leisten, mit anderen Worten, einen besonderen Beitrag in der Ausbildung eben jener ,Vor-Urteile“ zur Kategorisierung und Bewertung unserer Umwelt (vgl. Carney 2003a: 212-213).

Dass vor allem urbane Räume immer wieder sowohl in sogenannten ,hochkulturellen' als auch ,populärkulturellen' Formen und Medien be- und verarbeitet worden sind, mag sicherlich daran liegen, dass die Komplexität der Großstadt sowohl auf Kulturschaffende als auch auf Rezipienten eine bisher ungebrochene Faszination ausgeübt hat. Seien es Juvenals satirische Inszenierungen Roms, seien es die Stadtdichtungen der Romantiker oder die großen Stadtromane des 20. Jahrhunderts, wie James Joyce' Ulysses, John Dos Passos' Manhattan Transfer oder Alfred Döblins Berlin Alexanderplatz, seien es Filme oder Fernsehserien wie Friends oder Sex in the City, Comics wie Spiderman oder Popsongs: All diese Formate inszenieren urbane Räume auf jeweils medienund genrespezifische Art und Weise. Sie beziehen (bewusst oder unbewusst, intendiert oder nicht) Position zu diesen Räumen und bieten ihren ,Nutzern“ durch die Bereitstellung von Wahrnehmungs- und Deutungsmustern normative Ausgangspunkte zur eigenen Bewertung eben dieser Räume.

\section{Die diskursive Inszenierung New Yorks im Popsong: „Empire State of Mind“ von Jay-Z und Alicia Keys}

Wie diese mediale und generische Vor-Formatierung genau geschieht, möchten wir im Folgenden anhand der Inszenierung New Yorks in der Populärkultur deutlich machen. Da in diesem Beitrag aus Platzgründen eine systematische Aufarbeitung der unterschiedlichen Formen und Funktionen der populärkulturellen Repräsentationen New Yorks nicht möglich ist, haben wir uns an dieser 
Stelle für die Betrachtung nur eines Mediums entschieden, die zumindest andeuten soll, welche Ressourcen Populärkultur zur Bewertung urbaner Räume bereitzustellen vermag. Genauer: Wir werden uns einem Popsong über New York zuwenden - nicht zuletzt deshalb, da die Großstadt nicht nur für die Entwicklung unterschiedlicher musikalischer Genres wesentlich war und ist (man denke nur an solch spezifisch urbane Musikkulturen wie Hip-Hop), sondern auch, weil die Großstadt bzw. das Großstadtleben eines der zentralen Themen im Popsong zu sein scheint: „The city has become one of the most important musical places [...], both in origin of music genres/subgenres and lyrical content“, stellt beispielsweise George O. Carney fest und fügt hinzu: „New York City dominated city song titles during the early twentieth century with ,In Good Old New York Town“, ,Broadway Melody‘ and ,Manhattan Serenade“ as prominent examples“ (2003: 208). Es ließen sich zahlreiche weitere Titel nennen, die in den folgenden Jahrzehnten und auch in der zweiten Hälfte des 20. Jahrhunderts zu Klassikern geworden sind, allen voran vielleicht Frank Sinatras „New York, New York“ - wer sich genauer informieren möchte, werfe einen Blick in die beeindruckend lange Titelliste von New-York-Songs auf Wikipedia. ${ }^{4}$

Selbstredend ist die Inszenierung New Yorks in diesen unzähligen Songs nicht einheitlich und abhängig von unterschiedlichen Zielsetzungen - die Darstellung der Stadt als unbedingtem ,Erfüllungsort“ des American Dream in Sinatras Lied wird man im dezidiert politisch motivierten Rap der 1980er Jahre, der eben auch in New York angesiedelt war (vgl. Carney 2003b: 98-99), so nicht unbedingt wiederfinden. Aber auch hier ist die Verbindung der Stadt mit einem gewissen ,mindset‘, einer Grundeinstellung, eines bestimmten ,way of life‘ zu spüren. Mit anderen Worten: New York ist in den Songs über die Stadt mehr als nur Megacity; der ,Big Apple‘ wird vielmehr zum Signifikat bzw. zur Chiffre der uramerikanischen Idee der Selbstfindung und -verwirklichung. Und selbst wenn diese Selbstverwirklichung (wie im politischen Rap der 1980er Jahre oftmals thematisiert) aufgrund widriger Umstände nicht realisiert werden kann, bleibt allein die Möglichkeit dazu der Bezugsrahmen.

Ein Beispiel, in dem diese Verquickung von urbanem Raum und amerikanischem ,mindset“ bereits im Titel angekündigt wird, ist der Song „Empire State of Mind“ des Rappers Jay-Z und der R\&B-Sängerin Alicia Keys aus dem Jahr 2009. Wir gehen im Folgenden etwas näher auf diesen Song ein, um zu verdeutlichen, mit welchen Strategien hier eine ,mental map“ New Yorks etabliert wird, die sowohl als bewertende Praxis als auch als Grundlage für die Bewertung der

4 Vgl.http://en.wikipedia.org/wiki/List_of_songs_about_New_York_City; $<10.5 .2012>$. 
Stadt durch die Zuhörerinnen und Zuhörer fungieren mag. Wir beziehen uns in unserer Analyse des Songs auf das Musikvideo zu dem Song; durch eine Vielzahl von Verweisen auf ikonische und stark assoziationsbesetze Gebäude und Orte in Manhattan erweitert dieses die ,Kartierung، und Bewertung des urbanen Raums New York durch eine visuelle Dimension. ${ }^{5}$

Wie hier zunächst auf der visuellen Ebene deutlich wird, ruft der Clip in einer schnappschussartigen Sequenz eine Reihe ikonischer Elemente auf, die unmittelbar mit dem urbanen Raum New York in Verbindung gebracht werden, u.a. die zahllosen Wolkenkratzer (unter ihnen das Rockefeller Center und das Empire State Building), die ,yellow cabs‘, die Wall Street, das Yankee-Stadion, die U-Bahn, die Skyline des Financial District, den Times Square usw. Diese Form der Visualisierung markiert einerseits die Einzigartigkeit und Unverwechselbarkeit der Stadt und dient andererseits - und im wahrsten Sinne des Wortes - der ,Verortung' bzw. Selbstverortung der Interpreten. New York wird hier also einerseits ,kartiert` durch die Bereitstellung visueller Schlüsselkoordinaten, die durch ihre ständige mediale Wiederholung bereits integraler Bestandteil des kulturellen Wissens über diese Stadt sind, womit der Clip selbst auch zur Perpetuierung dieser Ikonographie der Metropole beiträgt und den Rezipienten so ein Wahrnehmungsmuster zur Verfügung stellt. Andererseits wird New York City in dem Clip als ,Bühne‘ für die Hip-Hop-Künstler konstituiert, die sich den ,kartierten' urbanen Raum als subkulturellen Raum mit dem Ziel der Selbstinszenierung performativ zu eigen machen.

Auf der Textebene wird dieses Muster ebenfalls produziert, hier durch den Rückgriff auf stereotype Konzeptionalisierungen von New York als „Concrete jungle where dreams are made of“ oder als „melting pot“, dessen Straßen und Lichter inspirierend wirken. Gleich zu Beginn des Songs heißt es:

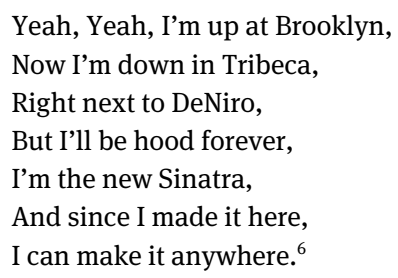

5 Vgl. www.youtube.com/watch?v=0UjsXo916I8; <10.5.2012>. Mit Stand vom 10.5.2012 verzeichnet das Video fast 136.000.000 clicks auf youtube.com und zählt damit zu den meistgesehenen Musikvideos.

6 Wir zitieren nach dem genannten Musikvideo. 
Interessanterweise mischen sich hier zur Etablierung eines diskursiv konstituierten Koordinatensystems Referenzen auf geographische Orte ("up at Brooklyn”, “down in Tribeca”) und ein Verweis auf eine der vielleicht bekanntesten medialen Repräsentationen New Yorks (gemeint ist die Anspielung auf Sinatras „New York, New York“), ohne dass dabei die subkulturelle Dimension des HipHop aufgegeben würde: Mit dem Satz „but I'll be hood forever“ nämlich verweist der Sprecher in durchaus mehrdeutiger Weise auf seine lokale wie subkulturelle Identität als in der Nachbarschaft Verwurzelter wie als Krimineller (so kann „hood“ hier sowohl „neighbourhood“ wie „Gangster“ bedeuten (vgl. die im OED belegten Bedeutungen 2) und 3)). Die (Selbst)Verortung im urbanen Raum wird also komplementiert durch eine (Selbst)Verortung in einer langen Tradition der Repräsentationen eben dieses Raumes; der Song wird an dieser Stelle auch zum Kommentar über die Medialität oder Medialisierung, des ,Big Apple‘. An anderer Stelle wiederum wird auf ähnlich metareferentielle Weise auf den urbanen Charakter des eigenen Genres hingewiesen, das seinen Ursprung dem Sprecher zufolge in New York gehabt habe:

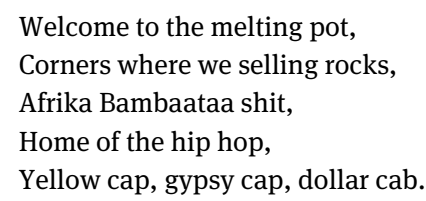

Diese medial vermittelte Bewertung New Yorks als quasi-mythischer Ort voller Energie und Kreativität erlebt insbesondere nach den Anschlägen auf das World Trade Center, die sicherlich auch aufgrund des zuvor beschriebenen symbolischen Stellenwerts der Stadt so traumatisierend wirkten, eine Renaissance. Der urbane Raum des ,Big Apple‘ wird einmal mehr - und vielleicht stärker als je zuvor - zum Sinnbild der amerikanischen Idee vom ,self-made man', dem ,new Sinatra', der seinen eigenen Weg besingt und letztlich auch zu gehen imstande ist ("And since I made it here / I can make it anywhere"). Und selbst wenn der Traum der Selbstverwirklichung nicht für alle in Erfüllung gehen wird - auch das wird nämlich besungen - steht man doch am Ende vereint für diese Idee ein, die an ebendiesem Tag im September 2001 in Frage gestellt wurde:

Cities is a pity half of y'all won't make it,

(...)

Labor Day parade, rest in peace Bob Marley,

Statue of Liberty, long live the World Trade,

Long live the king yo, I'm from the Empire State 

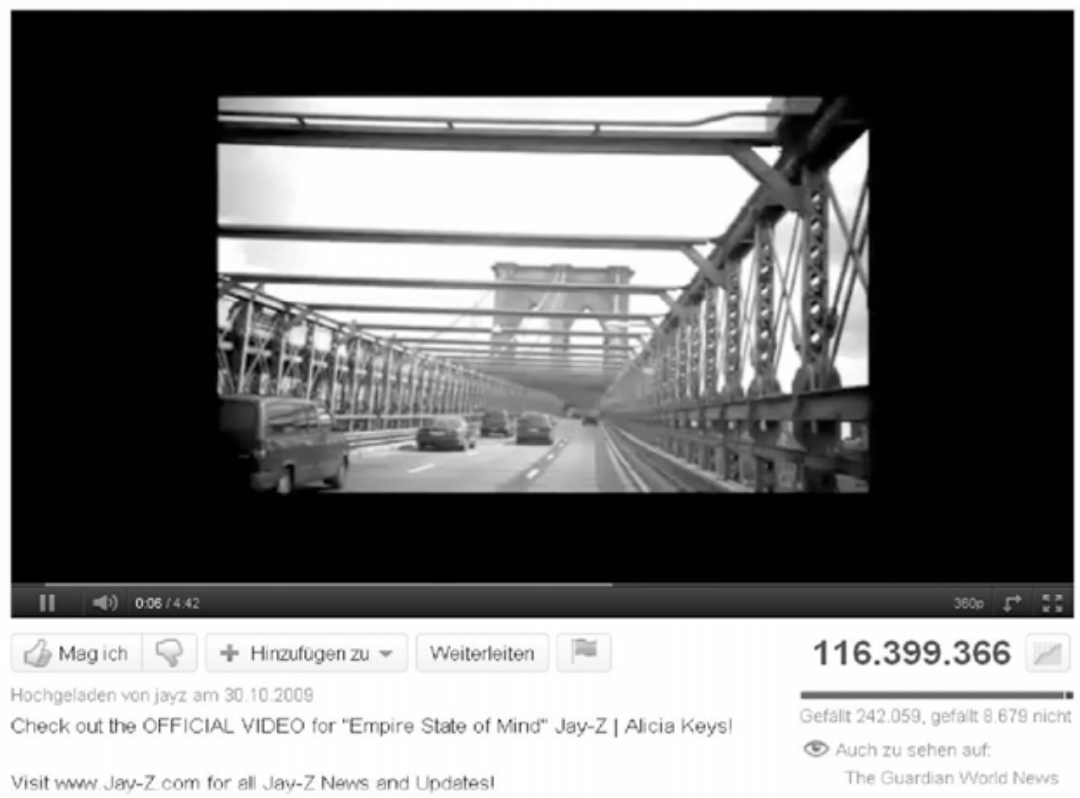

Weitere anzeigen $\approx$

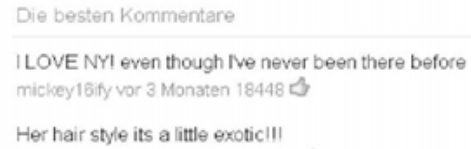

Abb. 1: Screenshot-Ausschnitt der YouTube-Seite mit Einstellung aus dem Video und Anfang des Kommentarfeldes (www.youtube.com/watch?v=0UjsXo91618; <27.10.2011>)

Dass der Clip auf diese Weise nicht nur eine Praktik der Bewertung des urbanen Raums von New York City darstellt, sondern auch Grundlage für die Bewertungen seiner ,User' sein kann, veranschaulicht eindrucksvoll die Diskussion des Musikvideos auf YouTube. Hier heißt es gleich in dem am häufigsten kommentierten Beitrag: „I LOVE NY! Even though I've never been there before“ (s. Abb. 1) - treffender lässt sich die zuvor beschriebene mediale Präfiguration urbaner Räume und deren normative Dimension schwerlich auf den Punkt bringen. ${ }^{7}$

7 Die romantisch verklärte Huldigung des urbanen Raums in „Empire State of Mind“ hat nicht nur im Forum von YouTube für einen enormen Nachhall gesorgt. Der Song war lange in den Charts und wurde auf verschiedenste Art und Weise zu einer Art „Erinnerungsstück“ an den 11. September. Wohl eine der bekanntesten Verarbeitungen des Liedes findet sich in einem kurzen 


\section{London umdeuten: Street Art als Taktik der performativen Aneignung und Bewertung urbaner Räume}

Eine andere Form der Bewertung urbaner Räume in und durch Populärkultur, die unmittelbar mit diesen Räumen verbunden ist, sind verschiedene Spielarten der Street Art, auf die wir im Folgenden anhand eines Beispiels aus dem angelsächsischen Kontext näher eingehen wollen. Street Art lässt sich - vor dem Hintergrund der Fragestellungen dieses Bandes - als performative Praxis der Aneignung bzw. Umdeutung oder Umbewertung urbaner Räume verstehen (vgl. etwa Gurr/Butler 2013). Einem solchen Verständnis von Street Art liegt die Beobachtung zugrunde, dass urbane Räume stets durch vorgegebene infrastrukturelle, architektonische und technologische Parameter definiert sind, durch die soziale Interaktion und Kommunikation nachhaltig beeinflusst und gesteuert werden kann. Diesen begrenzenden Systemeigenschaften, die sich als hegemoniale Strategien der Regulierung und Steuerung verstehen lassen, stehen allerdings eine Reihe kultureller Aneignungs- und Nutzungsformen gegenüber, durch welche die Funktionalität eben solcher Räume neu definiert und (auch) subversiven Zwecken, wie z.B. der Hinterfragung dominanter Sinnstiftungsmodelle und der damit einhergehenden Infragestellung etablierter Deutungshoheiten, dienlich gemacht werden kann. Zu diesen ,Taktiken' - die Michel de Certeau als die „clever tricks of the ,weak“" bezeichnet hat (1984: 40) -, die einzelne Akteure gemäß ihrer je individuellen Bedürfnisstruktur sowie unter Berücksichtigung von (potentiellen) Gruppenzugehörigkeiten als Teil eines individuellen Kommunikationsstils entwickeln, gehören auch solche kulturellen Ausdrucksformen, die im kreativen Umgang mit den durch die urbane Lebenswelt bereitgestellten Räumen eben diese Räume neu definieren und damit neu bewerten.

Ein Beispiel für diese Umdeutung oder Umbewertung urbaner Räume ist das Werk des Londoner Street Art-Künstlers Banksy, der längst nicht mehr nur in Insider-Kreisen ein Begriff ist. Insbesondere seine ,Kampagne“ gegen die zunehmende Überwachung und Kontrolle des öffentlichen Raums mag verdeutlichen, wie dem urbanen Raum sowie der strategischen Regulierung dieses Raums ,von oben“ hier eine neue Ordnung gegenübergestellt wird und wie dieser sozusagen als ,Protestraum‘ neu definiert wird. Repräsentativ und ein be-

TV-Spot zur Ehrung der New Yorker Feuerwehr des afro-amerikanischen Filmemachers und Drehbuchautors Spike Lee (vgl. http://www.youtube.com/watch?v=ZNbC-9jMJn4; <10.5.2012>). 
sonders eindrückliches Beispiel ist der von ihm in einer klandestin-logistischen Großaktion inklusive Auf- und Abbau eines mehrstöckigen Baugerüsts angebrachte großformative Schriftzug „One Nation under CCTV“ unmittelbar im Sichtfeld einer Überwachungskamera (vgl. Moore 2008). Diese Intervention bezieht sich nicht nur kritisch auf die genannte Tendenz zur flächendeckenden Überwachung des Stadtraums, für die gerade London bekannt ist, sondern bezieht einen erheblichen Teil ihrer subversive Energie gerade aus der direkten Präsenz eben jener technischen Infrastruktur, die sie so unmittelbar kritisiert. ${ }^{8}$

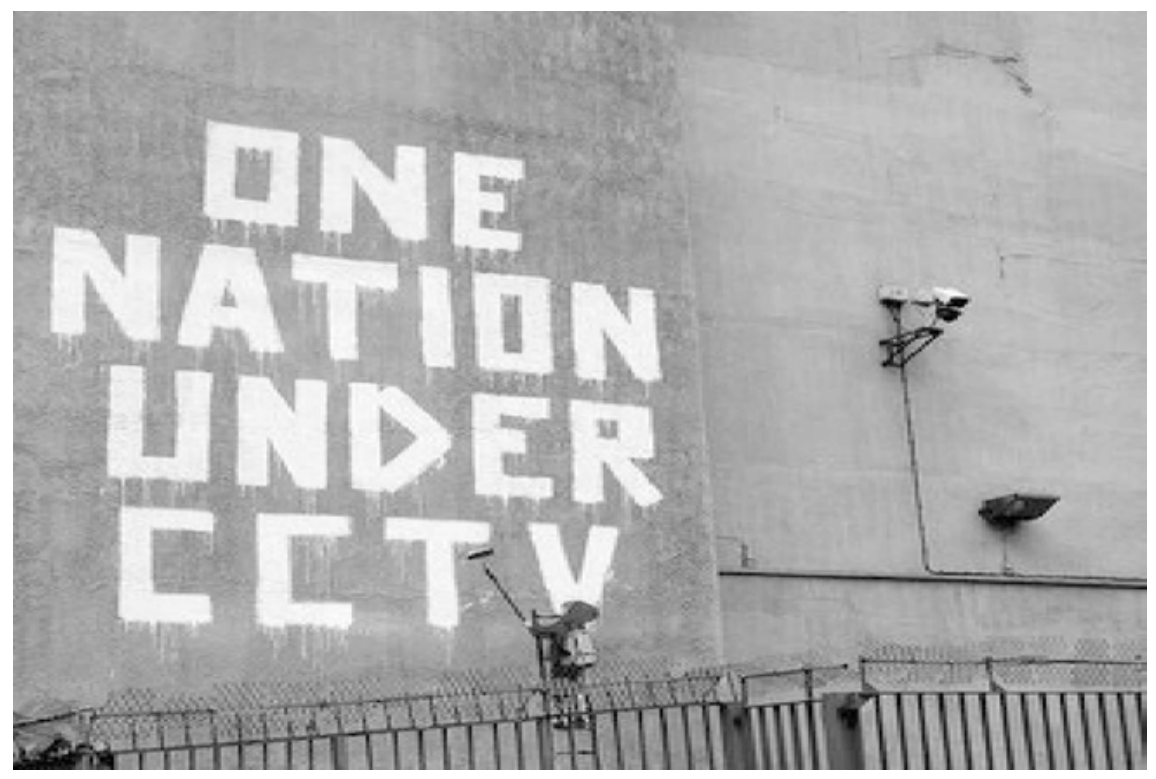

Abb. 2: Quelle: Banksy. Wall and Piece. London: Century, 2006

Banksys Werke sind hier einerseits populärkulturelle Ressource zur Umdeutung urbanen Raums; genauso ist aber der Raum Ressource für seine Werke. Die zuvor bereits angesprochene Unmittelbarkeit, also das Abhängigkeitsverhältnis zwischen urbanem Raum und urbaner Popkultur wird also besonders deutlich in dieser Form der performativen Aneignung der architektonischen Textur, die die Stadt ,zur Verfügung، stellt.

$8 \mathrm{Zu}$ nennen wären hier auch zahlreiche vergleichbare Schriftzüge und Installationen im Blickfeld von CCTV-Kameras, vgl. Banksy 2006: 86-89, 216-217. 
Die Bewertung urbaner Räume funktioniert also sowohl durch verschiedene Formen der diskursiven Inszenierung urbaner Räume, deren oft stark normative Dimension den Rezipienten Bewertungsmuster zur Verfügung stellt und damit die tatsächliche Bewertung urbaner Räume medial präfiguriert, als auch in Form von Praktiken der interagierenden Aneignung urbaner Räume, deren unmittelbare Umdeutung selbstredend auch Einfluss auf die Wahrnehmung und die Bewertung eben dieser Räume hat. Urbane Populärkultur in ihren verschiedenen Ausprägungen kann also - wie die Analyse unserer Beispiele veranschaulichen konnte - in der Tat sowohl Bewertungsressource als auch Bewertungspraxis sein.

\section{Zwischen Kunst und Kommerz: Die Bewertung urbaner Populärkultur als Distinktionspraxis zur Generierung eines ,urbanen Habitus“}

Gerade Banksys Werke - wie Street Art generell - haben längst ihre subkulturelle Nische verlassen und sind einer breiten Öffentlichkeit zugänglich gemacht worden. Dieses ,Mainstreaming‘ der Subkultur führte einerseits zu einer ,Vermarktung، von Banksy-Werken als touristische Ziele (vgl. etwa Bull 2008) und zu Wertsteigerungen von Banksy-Originalen auf dem Kunstmarkt, was die groteske Folge hatte, dass sich Kunstsammler aus aller Welt Teile von Londoner Hausfassaden in ihre Galerien stellten (vgl. hierzu insbesondere Reinecke 2007). Der Street Art-,Boom‘ löste darüber hinaus auch die Debatte um den Status dieser einst subkulturellen Ausdrucksform aus - haben wir es hier mit Kunst zu tun oder ist es am Ende doch nur Schmiererei?

Dass dieser Diskurs der Kommentierung und Bewertung urbaner Populärkultur mittlerweile selbst zu einer Praxis des Place-Making bzw. zum Ort der Aushandlung von Urbanität bzw. eines spezifisch ,urbanen“ Habitus geworden ist, soll der letzte Teil unseres Beitrag verdeutlichen, der sich einer (vermeintlichen) Dokumentation über Banksy mit dem Titel Exit Through the Gift Shop widmet. Es geht uns hier also nicht mehr um populärkulturelle Formen der Bewertung von Stadt als urbanem Raum, sondern um die Bewertung urbaner populärkultureller Ausdrucksformen als Distinktionspraxis individueller und kollektiver Akteure - Bewertungen übrigens, die durchaus im realen urbanen Raum stattfinden können, aber nicht notwendigerweise müssen.

Exit Through the Gift Shop erzählt die Geschichte des französischen Amateurfilmemachers Thierry Guetta, der beabsichtigt, eine Dokumentation über 
Street Art zu drehen, dabei mit allerlei Figuren aus der Szene in Kontakt kommt und sich letztlich auf die Suche nach Banksy macht, dem Street Art-Künstler, der seine wahre Identität nicht zu erkennen gibt. Guetta möchte also das Geheimnis um Banksy lüften und begegnet ihm schließlich auch. Dann nimmt der Film allerdings eine überraschende Wendung: Banksy - dem wir im Film als einem ,Phantom' mit verzerrter Erzählerstimme begegnen - berichtet nunmehr über Guetta, der - fasziniert und inspiriert von der Street Art, die ihm während seines Drehs vor die Kamera gekommen ist - nun selbst in die Szene einsteigt und in kürzester Zeit grotesk erfolgreich wird.

Man zweifelt als Zuschauer spätestens an dieser Stelle an der Ernsthaftigkeit des Films, der in der zweiten Hälfte eine maßlose Überspitzung der Dynamik inszeniert, die Banksy und anderen Street Art-Künstlern zu einer immensen Popularität verholfen haben. Denn die ironische Brechung in der Darstellung des kometenhaften Aufstiegs von Guetta ist kaum zu übersehen bzw. überhören: Guetta entwickelt auffällig schnell Starallüren, wird zum Exzentriker und umgibt sich gerne mit einer Reihe von Celebrities. Möglicherweise liegt aber gerade in dieser ironischen Brechung das ernste Anliegen des Films, der zumindest in diesem zweiten Teil ganz deutlich macht, wie das ,Reden über urbane Populärkultur` einerseits den Status eben solcher populärkulturellen Praktiken und Ausdrucksformen wie Street Art beeinflusst (Stichwort: Kanonisierung) und andererseits die Positionen der ,redenden' Akteure im künstlerischen ,Feld“ bestimmt. Der Film thematisiert also hier den Diskurs über Street Art als Distinktionsmechanismus für individuelle und kollektive Akteure; gleichzeitig nimmt er - als mediale Inszenierung dieses Diskurses - eine Position in diesem Diskurs ein.

Die Kommunikation über Street Art (sowohl die im Film inszenierte als auch die durch den Film betriebene) und die darin verhandelten Positionen werden also - so unsere These - zur Grundlage der Ausbildung eines spezifisch ,urbanen'Verhaltensmusters. Mit anderen Worten: Ob ich ,urban' bin oder nicht bzw. mich ,urban' verhalte oder nicht, hängt also auch davon ab, wie ich urbane Populärkultur bewerte. Allerdings - und dies bringt uns am Ende erneut zurück zu der anfangs eingeführten Kategorie der ,Mittelbarkeit' - ist dieser Bewertungsdiskurs - wie oben bereits angedeutet - weitestgehend losgelöst vom urbanen Raum der Stadt.

,Urbanität‘ nämlich, wie sie sich innerhalb dieses Bewertungsdiskurses konstituiert, möchten wir eben gerade nicht als Kategorie zur Beschreibung der den urbanen Raum konstituierenden Parameter verstanden wissen. Vielmehr liegt es nahe, Urbanität hier als ein diskursiv generiertes Set von spezifischen, eben als ,urban' zu charakterisierenden Eigenschaften und Verhaltensweisen zu modellieren, das wiederum als Distinktionsmechanismus Prozesse der sozialen 
Abgrenzung und Identifikation organisiert. Urbanitätsdiskurse in urbaner Populärkultur und über urbane Populärkultur werden so als Praktiken des PlaceMaking erkennbar, die über die vielfach gar nicht mehr notwendige Referenz auf einen lokalisierbaren Ort hinaus mindestens ebenso sehr einen Ort im Diskurs markieren. Vor dem Hintergrund dieses Begriffsverständnisses ist die Bewertung urbaner populärkultureller Ausdrucksformen dann in der Tat nicht mehr an den Ort der Stadt gebunden, sondern findet in einem vom physischen Raum der Stadt losgelösten ,diskursiven Raum' statt. Bewertung ist also nicht nur „ein ausgesprochen verbreiteter Modus in der Kommunikation über Stadt“, wie es in der Kurzbeschreibung des diesem Band zugrundeliegenden Symposions hieß, sondern auch ein ebenso verbreiteter Modus in der Kommunikation über Urbanität, die wir als diskursiv konstituierte Distinktionskategorie verstehen, die mit dem urbanen Raum der Stadt zwar in der Regel assoziiert ist, aber nicht notwendigerweise daran gebunden sein muss. ${ }^{9}$

Urbane Populärkultur, so unser Fazit, stellt ihren Rezipienten verschiedene Muster zur Wahrnehmung und Bewertung urbaner Räume zur Verfügung und fungiert so - mit zunehmender Medialisierung zumindest potentiell vom physi-

\footnotetext{
9 Ein solches Verständnis von Urbanität wird der Diskussion um die vielfach postulierte Enträumlichung im Zuge technologischer Entwicklungen (vgl. etwa Castells 1996) und der vielfach diagnostizierten Ubiquität des Urbanen bzw. der Auflösung des Stadt-LandGegensatzes (vgl. etwa Wirth 1964, Siebel 2004, Hassenpflug 2006) ebenso gerecht wie den historisch und kulturell jeweils verschiedenen Urbanitätsvorstellungen, etwa jeweils anderen Auffassungen vom Verhältnis von Privatheit und Öffentlichkeit (vgl. etwa Hassenpflug 2010). Zwar ist die Entkopplung von Stadt und Urbanität in neuerer Zeit bereits verschiedentlich diskutiert worden. Dabei ist die etwa von Dierksmeier im Anschluss an eine fundierte Kritik etablierter Urbanitätskonzepte entwickelte Vorstellung, die gerade dazu dienen soll, Urbanität unabhängig vom physischen Ort der Stadt zu fassen, zwar mit der Vorstellung eines spezifisch urbanen Habitus und der Definition von Urbanität als „Folge der Ausdifferenzierung und Pluralisierung von Lebensformen“ (Dierksmeier 2009: 70) in Bezug auf das hier formulierte Urbanitätsverständnis anschlussfähig; allerdings ist sie insofern problematisch, als sie die „bestimmte strukturelle Fremdheit der Städter“ (ebd.) und den damit einhergehenden Individualisierungsdruck als Ursache dieser Pluralisierung sieht und damit den Akzent lediglich vom physischen Ort der Stadt „hin zum Stadtbewohner“ (81) verschiebt und so der Engführung von Urbanitätsbegriff und Stadt nicht entgeht. Zudem vertritt Dierksmeier (wie auch andere Ansätze der Urbanitätsforschung) die Ansicht, das ,Wesen“ der Urbanität lasse sich in einer distinkten Reihe von Verhaltensmerkmalen beschreiben. Das hier skizzierte Verständnis unterscheidet sich von diesen Ansätzen insbesondere dadurch, dass es Urbanität nicht als weitgehend statische, bestimmten Akteuren entweder zugeschriebene oder abgesprochene Eigenschaft beschreibt, sondern von der Diskursivität des Urbanen ausgeht und vor diesem Hintergrund vielmehr die komplexen kommunikativen Prozesse der Konstruktion und Verhandlung von Urbanität, die sich je nach historischem und kulturellem Kontext voneinander unterscheiden, in den Blick nimmt.
} 
schen Raum der Stadt entkoppelt - als Anlass und Ressource zur Affirmation oder Revision etablierter oder emergenter Urbanitätskonzeptionen. Denn neben der performativ-aneignenden ,Nutzung ' der gegebenen urbanen Infrastruktur bilden die hier untersuchten Praktiken urbaner Kommunikation auch immer ein Moment der diskursiven Inszenierung und Repräsentation des urbanen Raums und lassen sich damit gleichermaßen als Formen der Kommunikation über Stadt und Urbanität verstehen. Unsere Analyse urbaner Populärkultur als Praxis und Ressource der Bewertung urbaner Räume versucht so zudem einen Beitrag zur ebenso aktuellen wie relevanten Frage nach einem zeitgemäßen Urbanitätsverständnis zu leisten - einem Verständnis, das die kommunikativen Parameter der Konstitution von Urbanität identifiziert und auf diese Weise über essentialistische Konzepte von Urbanität hinausgeht.

\section{Literaturverzeichnis}

\section{Forschungsliteratur}

Bourdieu Pierre (1989): „Antworten auf einige Einwände“. In: Klaus Eder (Hg.): Klassenlage, Lebensstil und kulturelle Praxis. Beiträge zur Auseinandersetzung mit Pierre Bourdieus Klassentheorie. Frankfurt am Main: Suhrkamp, 395-410.

Carney, George 0. ( $\left.{ }^{4} 2003 a\right)$ : „Music and Place“. In: George O. Carney (Hg.): The Sounds of People and Places. A Geography of American Music from Country to Classical and Blues to Bop. Lanham: Rowman \& Littlefield, 203-216.

Carney, George 0. ( $\left.{ }^{4} 2003 b\right):$ „Rappin' in America: A Regional Music Phenomenon“. In: George 0 . Carney (Hg.): The Sounds of People and Places. A Geography of American Music from Country to Classical and Blues to Bop. Lanham: Rowman \& Littlefield, 93-118.

Castells, Manuel (1996): The Information Age. Economy, Society and Culture. Vol. I: The Rise of the Network Society. Oxford: Blackwell.

de Certeau, Michel (1984): The Practice of Everday Life. Übers. Steven Rendall. Berkeley: University of California Press [Arts de faire. Paris: Union Générale d'Éditions, 1980].

Dierksmeier, Peter (2009): Urbanität als Habitus. Zur Sozialgeographie städtischen Lebens auf dem Land. Bielefeld: transcript.

Gould, Peter (1966): „On Mental Maps“. Discussion Paper No. 9. Michigan Interuniversity Community of Mathematical Geographers. Ann Arbor: University of Michigan.

Gould, Peter \& Rodney White (1974): Mental Maps. Harmondsworth: Penguin.

Grossberg, Lawrence (1992). We Gotta Get Out of This Place: Popular Conservatism and Postmodern Culture. New York: Routledge.

Grosz, Elisabeth (1995): „Bodies - Cities“. In: Elisabeth Grosz (Hg.): Space, Time, and Perversion. Essays on the Politics of Bodies. New York: Routledge, 103-110.

Gurr, Jens Martin (2010): „Urbanity, Urban Culture and the European Metropolis“. In: Michael Szczekalla (Hg.): Britannien und Europa - Studien zur Literatur-, Geistes- und Kulturgeschichte. Festschrift für Jürgen Klein. Frankfurt/Main: Lang, 241-255. 
Gurr, Jens Martin \& Martin Butler (2011): „Against the ,Erasure of Memory“ in Los Angeles City Planning. Strategies of Re-Ethnicizing L.A. in Digital Fiction“. In: Olaf Kaltmeier (Hg.): Selling EthniCity. Urban Cultural Politics in the Americas. London: Ashgate, 145-163.

Gurr, Jens Martin \& Martin Butler (2013). „On the ,Cultural Dimension of Sustainability“ in Urban Systems: Urban Cultures as Ecological ,Force-Fields‘ in Processes of Sustainable Development“. In: Stefanie Caeners, Michael Eisinger, Jens Martin Gurr, J. Alexander Schmidt (Hgg.): Healthy and Liveable Cities: Selected Papers from the Essen Conference / Gesunde und lebenswerte Städte: Ausgewählte Beiträge der Essener Tagung. Ludwigsburg: avedition, 138-151.

Hassenpflug, Dieter (2006): „Urbanität“. In: Dieter Hassenpflug (Hg.): Reflexive Urbanistik. Reden und Aufsätze zur europäischen Stadt. Weimar: Verlag der Bauhaus-Universität, 57-68.

Hassenpflug, Dieter (2010): The Urban Code of China. Basel: Birkhäuser.

Lynch, Kevin (1960): The Image of the City. Cambridge, MA: MIT Press.

Reinecke, Julia (2007): Street-Art. Eine Subkultur zwischen Kunst und Kommerz. Bielefeld: transcript.

Sennett, Richard (1974): Flesh and Stone. The Body and the City in Western Civilization. New York: Norton.

Siebel, Walter (2004): „Einleitung. Die europäische Stadt.“ In: Walter Siebel (Hg.): Die europäische Stadt. Frankfurt a.M.: Suhrkamp, 11-50.

Wirth, Louis (1956): „Rural-Urban Differences“. Nachdruck in: Albert J. Reiss, Jr. (Hg.). On Cities and Social Life. Chicago: University of Chicago Press, 221-225.

\section{Quellen}

Banksy (2006). Wall and Piece. London: Century.

Banksy (2010): Exit Through the Gift Shop. Paranoid Pictures [Film].

Bull, Martin (32008): Banksy Locations \& Tours. A Collection of Graffiti Locations and Photographs in London. London: shellshock publishing.

\section{Webnachweise}

http://en.wikipedia.org/wiki/List_of_songs_about_New_York_City; <12.5.2012>

Jay-Z \& Alicia Keys (2009). „Empire State of Mind“. [www.youtube.com/watch?v=0UjsXo91618]; $<12.5 .2012>$.

Moore, Matthew (2008): „Banksy pulls off daring CCTV protest in London“. The Telegraph, 15.5.2008. [www.telegraph.co.uk/news/uknews/1895625/Banksy-pulls-off-daring-CCTVprotest-in-London.html]; <12.5.2012〉.

\section{Abbildungsverzeichnis}

Abb. 1: Screenshot-Ausschnitt der YouTube-Seite mit Einstellung aus dem Video und Anfang des Kommentarfeldes

Abb. 2: Quelle: Banksy. Wall and Piece. London: Century, 2006 


\section{DuEPublico}

Duisburg-Essen Publications online
UNIVERSITÄT

DEUSS B B R R G

offen im Denken

Ub $\mid \begin{gathered}\text { universitäts } \\ \text { bibliothek }\end{gathered}$

Dieser Text wird über DuEPublico, dem Dokumenten- und Publikationsserver der Universität Duisburg-Essen, zur Verfügung gestellt. Die hier veröffentlichte Version der EPublikation kann von einer eventuell ebenfalls veröffentlichten Verlagsversion abweichen.

DOI: $\quad 10.1515 / 9783110365818.369$

URN: urn:nbn:de:hbz:464-20210312-095411-3

Butler, Martin and Gurr, Jens Martin. "Urbane Populärkultur als Bewertungspraxis und ressource". DOI: https://doi.org/10.1515/9783110365818.369.

In: Place-Making in urbanen Diskursen, edited by Ingo Warnke and Beatrix Busse, Berlin, München, Boston: De Gruyter, 2014, e-ISBN (PDF) 978-3-11-036581-8. DOI: https://doi.org/10.1515/9783110365818 pp. 369-384.

(C) 2014 Walter de Gruyter GmbH, Berlin/München/Boston Alle Rechte vorbehalten. 